\title{
Thrombopoietin Receptor Levels in Tumor Cell Lines and Primary Tumors
}

\section{Connie L. Erickson-Miller, ${ }^{1}$ Antony Chadderton, ${ }^{1}$ Anna Gibbard, ${ }^{2}$ Jennifer Kirchner, ${ }^{1}$ Kodandaram Pillarisetti, ${ }^{1}$ Katherine Baker, ${ }^{3}$ Lini Pandite, ${ }^{3}$ Iman El-Hariry, ${ }^{4}$ Yasser Mostafa Kamel, ${ }^{5}$ Yuan Liu, ${ }^{6}$ Anne-Marie Martin, ${ }^{5}$ and Conrad Messam ${ }^{7}$}

${ }^{1}$ Oncology Biology, GlaxoSmithKline, 1250 South Collegeville Road, Collegeville, PA 19426-2990, USA

${ }^{2}$ Oncology Biomarkers, GlaxoSmithKline, Morphotek, 210 Welsh Pool Road, Exton, PA 19341, USA

${ }^{3}$ Oncology Clinical, GlaxoSmithKline, 5 Moore Drive, Research Triangle Park, NC 27709, USA

${ }^{4}$ Oncology Biomarkers, GlaxoSmithKline, Astellas Pharmaceuticals, 3 Parkway North, Deerfield, IL 60015, USA

${ }^{5}$ Oncology Clinical, GlaxoSmithKline, Oncology R\&D, Building 11, 1-3 Bridge Road, Stockley Park West, Uxbridge, Middlesex UB11 1BT, UK

${ }^{6}$ Oncology Biomarkers, GlaxoSmithKline, 1250 South Collegeville Road, Collegeville, PA 19426-2990, USA

${ }^{7}$ Oncology Clinical, GlaxoSmithKline, 1250 South Collegeville Road, Collegeville, PA 19426-2990, USA

Correspondence should be addressed to Connie L. Erickson-Miller, erickc00@gsk.com

Received 3 June 2010; Accepted 24 September 2010

Academic Editor: Edgar Selzer

Copyright (C) 2010 Connie L. Erickson-Miller et al. This is an open access article distributed under the Creative Commons Attribution License, which permits unrestricted use, distribution, and reproduction in any medium, provided the original work is properly cited.

\begin{abstract}
Thrombopoietin (TPO) receptor agonists represent a new approach for the treatment of thrombocytopenia, which may develop as a consequence of immune thrombocytopenia, chemotherapy treatment, chronic hepatitis $\mathrm{C}$ infection, or myelodysplastic syndromes. There are concerns that use of certain growth factors can hasten disease progression in some types of hematologic malignancies and solid tumors. In this study, expression of MPL (TPO-R) mRNA was examined in tumor cell lines, patient tumor samples (renal cell carcinoma, prostatic carcinoma, soft tissue and bony/cartilage sarcoma, colon cancer, and lymphoma), and normal tissues using microarray analysis and qRT-PCR. MPL mRNA is expressed at very low or undetectable levels compared with erythropoietin receptor (EPOR), human epidermal growth factor (ERBB2; HER2), and insulin-like growth factor-1 receptor $(I G F 1 R)$ in these patient samples. These data suggest TPO-R agonists will likely preferentially stimulate proliferation and differentiation of cells of megakaryocytic lineage, potentially demonstrating their utility for correcting thrombocytopenia in clinical settings.
\end{abstract}

\section{Introduction}

Thrombopoietin (TPO) is the key cytokine involved in the regulation of thrombopoiesis. Its activities are twofold: TPO stimulates both megakaryocyte colony formation and enhances megakaryocyte maturation. Megakaryocytes and their precursors possess the thrombopoietin receptor (TPO$\mathrm{R} ; \mathrm{MPL}$ ) on their surfaces. TPO is constitutively expressed, but the level of circulating TPO is directly related to platelet mass $[1-5]$. The JAK-STAT signal transduction pathway is activated upon binding of TPO to its receptor, resulting in changes in gene expression that promote megakaryocytic differentiation, increase megakaryocyte ploidy, and stimulate the release of platelets into the peripheral circulation.

Thrombocytopenia, defined as a platelet count less than $150,000 / \mu \mathrm{L}$, represents an important clinical issue because it can increase the risk for bleeding. Spontaneous bleeding can occur when platelet counts drop below $20,000 / \mu \mathrm{L}$ [6]. It develops as a result of an imbalance between platelet production and platelet consumption. Thrombocytopenia develops commonly in patients with a variety of conditions including HIV, chronic hepatitis C infection, portal hypertension, systemic lupus erythematosus, myelodysplastic syndromes, 
and cancer [7-11]. Thrombocytopenia can also result from myelosuppressive chemotherapy, although bleeding episodes are infrequent overall. Thrombocytopenia can result in dose delays and dose reductions of chemotherapy that could negatively impact disease control [11]. However, bleeding is associated with poor performance status, multiple comorbidities, bone marrow metastases, and low baseline platelet counts, and, when it occurs, bleeding predicts poor clinical outcomes [11]. Although development of recombinant TPO was discontinued because of neutralizing antibody formation, 2 TPO-R agonists, eltrombopag and romiplostim, have been approved for the treatment of chronic immune thrombocytopenic purpura (ITP). These TPO-R agonists stimulate TPO-dependent cell lines via JAK-STAT signaling and demonstrate increased platelets in humans $[6,12-15]$.

Other growth factors have the potential to stimulate growth of tumors. A recent review article described the use of erythropoiesis-stimulating agents to increase hemoglobin levels in patients with cancer; numerous studies were identified in which use of these drugs increased the risk of treatment-associated death, perhaps through tumor progression [16, 17]. Erythropoietin receptor messenger ribonucleic acid (mRNA) and/or protein has been identified in a wide range of cancers, leading to the speculation that tumor progression may be a consequence of activation of endogenous erythropoietin receptors by exogenous erythropoiesis-stimulating agents, thereby promoting tumor cell proliferation and angiogenesis and inhibiting apoptosis. Acute myelogenous leukemia (AML) blast cells express $M P L$ mRNA and protein [18]. TPO stimulated blast colony formation in samples from approximately $50 \%$ of patients with AML in one study [19], and enhanced proliferation of a megakaryocytic leukemic cell line [20]. Results from other investigators confirmed this and demonstrated that TPO induced cell cycle activation and could protect AML blasts from programmed cell death [21]. Eltrombopag does not appear to stimulate proliferation of AML/myelodysplastic syndromes (MDS) patient blood and marrow samples or nonmegakaryocytic leukemia and lymphoma cell lines [22-24]. In fact, there is a decrease in proliferation at physiologically achievable concentrations of eltrombopag. Although the intensity of signaling induced by eltrombopag is lower than that induced by TPO, a mechanism for this differential effect has not been identified $[14,22,23]$.

There is little quantitative information available on the expression of TPO-R on solid tumors. In a recent examination of breast, nonsmall cell lung cancer (NSCLC), and ovarian tumor samples, very little, or moreoften, no MPL expression was detectable on these patient samples [25]. To further our knowledge of MPL mRNA expression, quantitative reverse transcription-polymerase chain reaction (qRT-PCR) and microarray analysis were performed on tumor cell lines and in tumor samples from renal cell carcinomas, prostate cancers, lymphomas, colon cancers, and sarcomas.

\section{Methods}

2.1. Tumor Cell Lines. Three hundred and fifty-five cell lines of various tissue types were obtained from the American Type Culture Collection (Rockville, MD, USA), the German Collection of Microorganisms and Cell Cultures (Braunschweig, Germany), the National Cancer Institute (Bethesda, MD, USA), and the European Collection of Cell Cultures (Salisbury, UK). All cells were in log phase growth at the time of collection. The complete list of tumor cell lines used in this study is provided in the Supplementary Material available online at doi: 10.1155/2010/135354.

2.2. Patient Tissue Samples. Colon (5 normal, 42 tumor), prostate ( 7 normal, 30 tumor, 11 benign hypertrophy), and lymphoma (3 normal spleen, 3 normal lymph nodes, 42 lymphoma) cDNA samples were obtained from Cytomyx LLC (Lexington, MA, USA). Archival tissue samples were also obtained from 105 patients with locally advanced or metastatic renal cell carcinoma (RCC) who failed, or who were unable to tolerate, first-line cytokine-based therapy. These patients were selected for epidermal growth factor receptor (EGFR) and/or ERBB2 positive $(1+, 2+$, or $3+)$ tumors. Archival tissue samples were obtained from an additional 46 patients who had metastatic or locally recurrent RCC of clear cell histology. These individuals either had no prior systemic therapy or had failed only 1 prior cytokinebased or bevacizumab-based therapy.

Additional archival tissue samples from 22 patients with soft tissue sarcomas (14 leiomyosarcomas, 8 malignant fibrous histiocytomas) and 12 bony/cartilage sarcomas (3 chondroblastomas, 7 osteosarcomas, 1 chordoma, 1 enchondroma) were obtained from the University of Pennsylvania (Philadelphia, PA, USA).

2.3. Microarray Analysis. Clinical tissue samples were all formalin fixed and paraffin embedded. RNA is often fragmented in these samples. To ensure the quality of RNA extraction, tumor tissues were microdissected using laser capture microdissection. RNA was rapidly extracted; 100 base pair (bp), $300 \mathrm{bp}$, and $400 \mathrm{bp}$ segments of the $\beta$-actin gene were tested using qRT-PCR. Samples with less than 32 cycle threshold $(\mathrm{Ct})$ at the $300 \mathrm{bp}$ level and less than $34 \mathrm{Ct}$ at the $400 \mathrm{bp}$ level were included in the microarray analysis.

Microarray analysis of all RCC samples was performed at Response Genetics, Inc. (Los Angeles, CA, USA) using the Affymetrix HG-U133 Plus2 chip. Microarray analysis was performed on the sarcoma tissue samples, using the Affymetrix HG-U133 chip, at the University of Pennsylvania. RNA microarray gene signal intensity was normalized using robust microarray average (RMA) analysis as described by Irizarry [26].

2.4. qRT-PCR Analysis. Primers and probes were customly made by Integrated DNA Technologies (Coralville, IA, USA). Sequences for the primers and probes used in this study are listed in the Supplementary Material. 
Cells from each cell line were lysed in Trizol reagent (Invitrogen). After phase separation with chloroform, total RNA was extracted using the RNeasy Mini Kit (Qiagen, Germantown, MD, USA) following the manufacturer's instructions. Genomic DNA contamination was removed using DNase I (Ambion, Austin, TX, USA). RNA samples were considered to be free of genomic DNA if no amplification was observed in a standard TaqMan assay using 10 ng of RNA and ACTB primer/probe oligonucleotides. The RNA was quantified using a Nanodrop analyzer and was subsequently converted to cDNA by reverse transcription utilizing the High Capacity cDNA Archive Kit (Applied Biosystems Inc., Foster City, CA, USA).

qRT-PCR was also performed on the cDNA from both the normal and tumor tissue samples and from the cell lines to assess the levels of $M P L$, erythropoietin receptor (EPOR), human epidermal growth factor (ERBB2; HER2), and insulin-like growth factor-1 receptor (IGF1R). Data were normalized to a set of housekeeping genes used as internal standards including GADPH, PPIA (cyclophilin-A), and $A C T B$ ( $\beta$-actin). The equivalent of $10 \mathrm{ng}$ mRNA per well was arrayed into 384-well plates using a Biomek FX robot (Beckman Coulter, Inc., Fullerton, CA, USA) and qRTPCR was carried out using a 7900HT Sequence Detector System (Applied Biosystems Inc.) in a $5 \mu \mathrm{L}$ reaction volume. TaqMan Universal PCR Master Mix 2X (Applied Biosystems Inc.) and universal PCR conditions recommended by the manufacturer were followed.

$\mathrm{Ct}$ values were analyzed using in-house analysis software in which values were normalized to the internal housekeeping genes in the reaction. Only samples with sufficient housekeeping gene expression $(\mathrm{Ct}<32)$ were used. Abundance was calculated using the formula: abundance $=$ $10 \mathrm{e}((40-\mathrm{Ct}) / 3.35)$. To normalize the data, samples were scaled relative to each other using the geometric mean of the set of valid housekeeping gene datapoints for that sample. Each datapoint was then expressed as the ratio of the housekeeping gene abundance in the sample to the average abundance of that housekeeping gene in all samples and marked invalid if it had statistically inconsistent behavior with the other housekeeping genes in those samples with similar tissue types.

\section{Results}

In most of the 355 tumor cell lines analyzed, MPL mRNA was expressed only at very low levels (Figure 1). Only 3 cell lines demonstrated expression of MPL mRNA in excess of 9500 normalized abundance, as illustrated in Figure 1. These were the erythroleukemia cell lines HEL 92.1.7 and KG-1 and the lung tumor cell line NCI-H510.

Table 1 summarizes normalized mRNA abundance for $M P L, E P O R, E R B B 2$, and IGF1R in all the cell lines studied. MPL expression was minimal. The mean normalized abundance for MPL mRNA was 1447 and the mode was 621. $E P O R$ mRNA, in contrast, was expressed at low to moderate levels (mean 12,587; mode 7811) and ERBB2 mRNA expression was markedly greater (mean 280,190 ; mode 40,828 ) as
TABLE 1: Normalized Abundance of MPL, EPOR, ERBB2, and IGF1R mRNA Across 355 Tumor Cell Line Types.

\begin{tabular}{lccccc}
\hline \multicolumn{5}{c}{ Cycle Threshold Values } \\
& Mode & Mean & SD & Minimum & Maximum \\
\hline MPL & 621 & 1447 & 463 & 1 & 172,402 \\
EPOR & 7811 & 12,587 & 1055 & 1 & 311,201 \\
ERBB2 & 40,828 & 280,190 & 63,578 & 197 & $12,632,200$ \\
IGF1R & 56,625 & 78,977 & 3914 & 1 & 535,859 \\
\hline
\end{tabular}

EPOR: erythropoietin receptor; $E R B B 2$ : human epidermal growth factor; $I G F 1 R$ : insulin growth factor-1 receptor; $M P L$ : thrombopoietin receptor; SD: standard deviation.

Cycle threshold values were calculated and samples normalized as described in the Materials and Methods section.

Table 2: Number (\%) of Primary Tumor Samples With Detectable MPL mRNA Expression Determined by Microarray Analysis.

\begin{tabular}{lccc}
\hline$n(\%)$ & $\begin{array}{c}\text { RCC } \\
n=151\end{array}$ & $\begin{array}{c}\text { Soft Tissue Sarcomas } \\
n=22\end{array}$ & $\begin{array}{c}\text { Bony/Cartilage } \\
\text { Sarcomas } \\
n=12\end{array}$ \\
\hline MPL & 0 & $7(32)$ & $4(33)$ \\
EPOR & $132(87)$ & $6(27)$ & $4(33)$ \\
ERBB2 & $122(81)$ & $21(95)$ & $9(75)$ \\
IGF1R & $81(54)$ & $\mathrm{ND}$ & $\mathrm{ND}$ \\
\hline
\end{tabular}

EPOR: erythropoietin receptor; ERBB2: human epidermal growth factor; IGF1R: insulin growth factor-1 receptor; $M P L$ : thrombopoietin receptor; ND: not determined; RCC: renal cell carcinoma; RMA: robust microarray averages. Detectable expression is determined by a RMA $\geq 50$.

was IGF1R mRNA expression mean 78,977; mode 56,625). The highest expression of ERBB2 was observed in breast tumor cell lines, as expected. ERBB2 and IGF1R mRNA demonstrated particularly higher expression than MPL or $E P O R$ in lung, brain, breast, and bladder cell lines, as shown in Figure 1.

Microarray data analysis of MPL mRNA expression was performed on primary tumor samples from RCC and soft tissue and bony/cartilage sarcomas. None of the 151 RCC tumors expressed MPL mRNA at detectable levels $($ RMA $\geq 50)$ (Figure 2, Table 2). However, 132 (87\%) showed detectable levels of EPOR mRNA, and 122 (81\%) demonstrated ERBB2 mRNA expression. Fewer RCC tumor samples $(81 ; 54 \%)$ expressed detectable IGF1R mRNA. $E P O R, E R B B 2$, and IGF1R mRNA levels were overall lower in these RCC samples than those observed in the breast and NSCLC tumors previously described [25].

Seven of $22(32 \%)$ soft tissue sarcomas and 4/12 bony/cartilage sarcomas (33\%) expressed MPL mRNA at detectable levels (Figure 2(b), Table 2). Similarly, 6/22 (27\%) soft tissue and 4/12 (33\%) bony/cartilage samples showed detectable levels of EPOR. The majority of samples (21/22 $(95 \%)$ and $9 / 12(75 \%)$, respectively) expressed detectable ERBB2 mRNA.

Microarray analysis is best used to describe relative differences in mRNA levels. qRT-PCR analysis was performed on additional samples to more quantitatively determine mRNA 

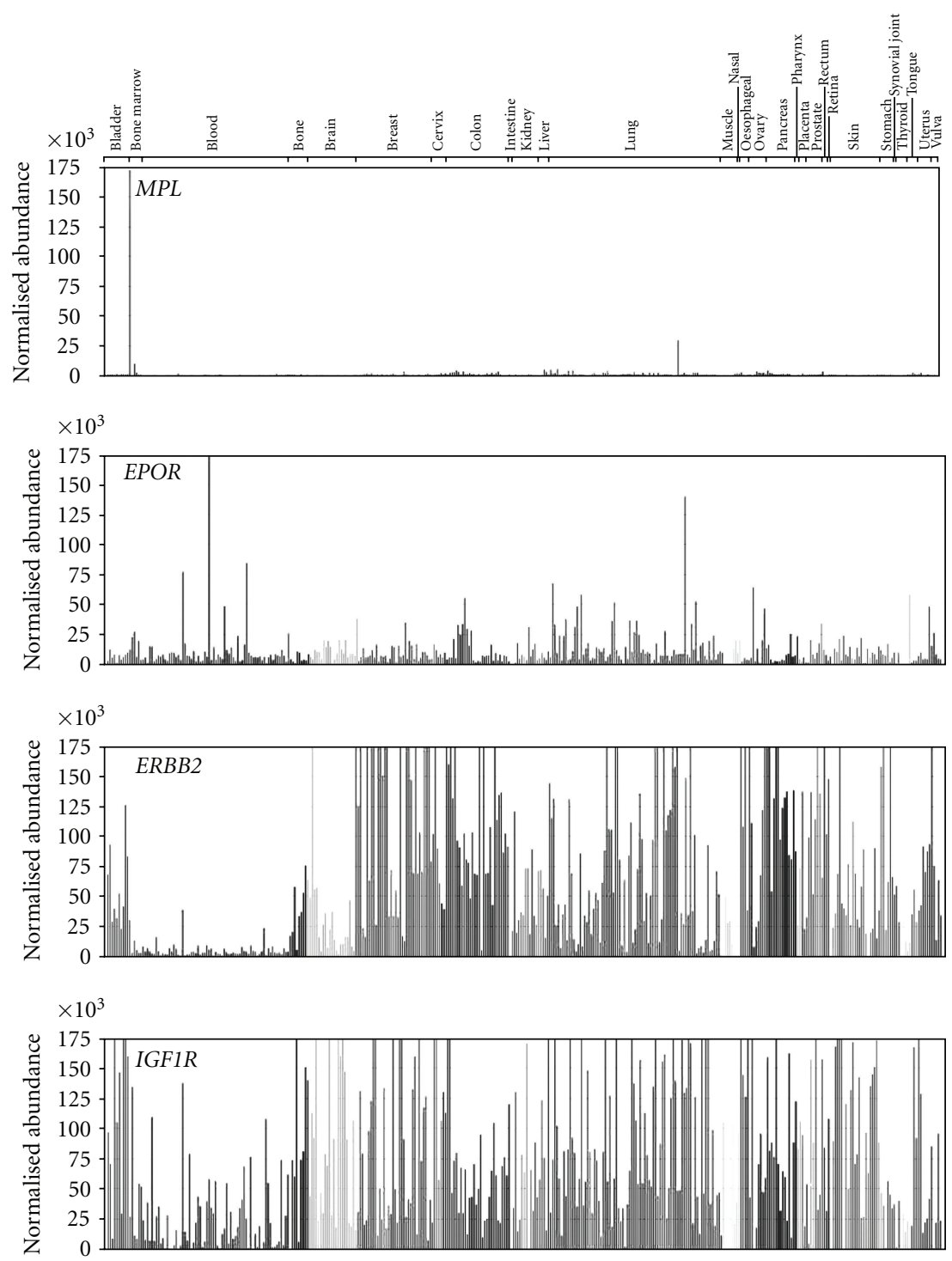

FIGURE 1: Quantitative reverse transcription-polymerase chain reaction measurement of $M P L, E P O R, E R B B 2$, and IGF1R mRNA in various tumor cell lines. Data were normalized to a set of housekeeping genes (GAPDH, cyclophilin, and $\beta$-actin). The normalized abundance of mRNA ( $y$-axis) is scaled to 175,000 for all samples. Cycle threshold $(\mathrm{Ct})$ values were calculated using the following equation: Abundance $=$ $10 \mathrm{e}((40-\mathrm{Ct}) / 3.35)$. Samples were scaled relative to each other using the geometric mean of the set of valid housekeeping gene datapoints for that sample.

expression in normal tissues and tumor samples. The qRTPCR data were normalized to a set of 3 housekeeping genes.

Among prostate samples, no normal or benign prostatic hypertrophy samples expressed detectable MPL mRNA whereas 1 tumor (3\%) expressed MPL (Table 3, Figure 3). All samples expressed detectable levels of EPOR and ERBB2. IGF1R mRNA expression was also common with 4/7 (57\%) normal prostate samples, 25/30 (83\%) prostate tumors, and 7/11 (64\%) benign prostatic hypertrophy samples expressing detectable levels.

None of the 6 normal lymph node and spleen samples or 42 lymphoma samples expressed detectable levels of $M P L$, ERBB2, or IGF1R mRNA (Table 3, Figure 3). In contrast, $3 / 6(50 \%)$ normal lymph node and spleen samples and
10/42 (24\%) lymphoma samples expressed detectable EPOR mRNA.

None of the 5 normal colon samples or 42 colon tumors expressed detectable MPL mRNA (Table 3, Figure 3). IGF1R mRNA expression was detectable in $1(20 \%)$ normal and 5 $(12 \%)$ adenocarcinoma of the colon samples. However, all of the normal colon samples and $27(64 \%)$ colon tumors expressed detectable EPOR mRNA and all samples expressed detectable ERBB2.

The stage of disease was available for the samples of lymphoma and colon and prostate tumors, however, due to the lack of detectable expression of MPL mRNA in these tumor types, no interpretation could be made on the relationships between expression levels and stage of disease. 
TABle 3: Number (\%) of Primary Tumor Samples With Detectable mRNA Expression Determined by qRT-PCR.

\begin{tabular}{|c|c|c|c|c|c|c|c|}
\hline$n(\%)$ & $\begin{array}{c}\text { Prostate Normal } \\
\quad n=7\end{array}$ & $\begin{array}{c}\text { Prostate Tumor } \\
\quad n=30\end{array}$ & $\begin{array}{c}\text { Benign Prostatic } \\
\text { Hypertrophy } \\
n=11\end{array}$ & $\begin{array}{c}\text { Normal Lymph } \\
\text { Node and Spleen } \\
n=6\end{array}$ & $\begin{array}{c}\text { Lymphoma } \\
n=42\end{array}$ & $\begin{array}{c}\text { Colon Normal } \\
n=5\end{array}$ & $\begin{array}{c}\text { Colon Tumor } \\
n=42\end{array}$ \\
\hline$M P L$ & 0 & $1(3)$ & 0 & 0 & 0 & 0 & 0 \\
\hline$E P O R$ & $7(100)$ & $30(100)$ & $11(100)$ & $3(50)$ & $10(24)$ & $5(100)$ & $27(64)$ \\
\hline$E R B B 2$ & $7(100)$ & $30(100)$ & $11(100)$ & 0 & 0 & $5(100)$ & $42(100)$ \\
\hline$I G F 1 R$ & $4(57)$ & $25(83)$ & $7(64)$ & 0 & 0 & $1(20)$ & $5(12)$ \\
\hline
\end{tabular}

EPOR: erythropoietin receptor; ERBB2: human epidermal growth factor; IGF1R: insulin growth factor-1 receptor; MPL: thrombopoietin receptor; qRT-PCR: quantitative reverse transcription-polymerase chain reaction.

Detectable expression is determined by a normalized abundance $\geq 50$.

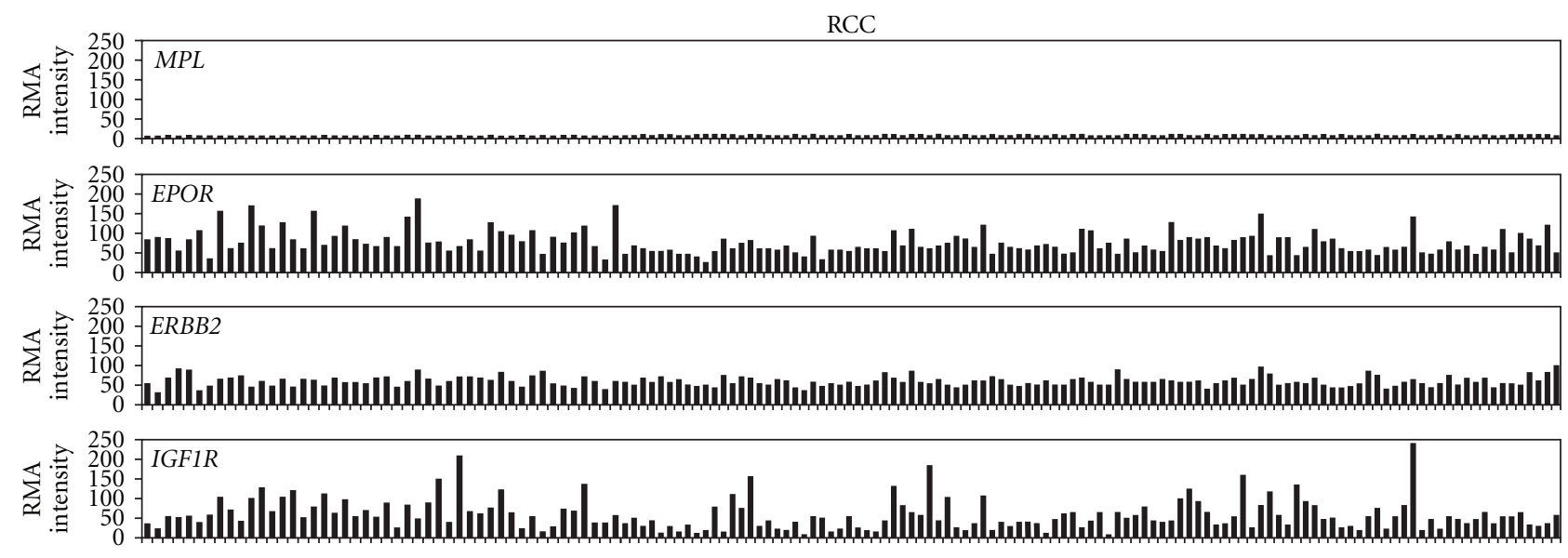

(a) Microarray data on primary renal cell carcinoma samples
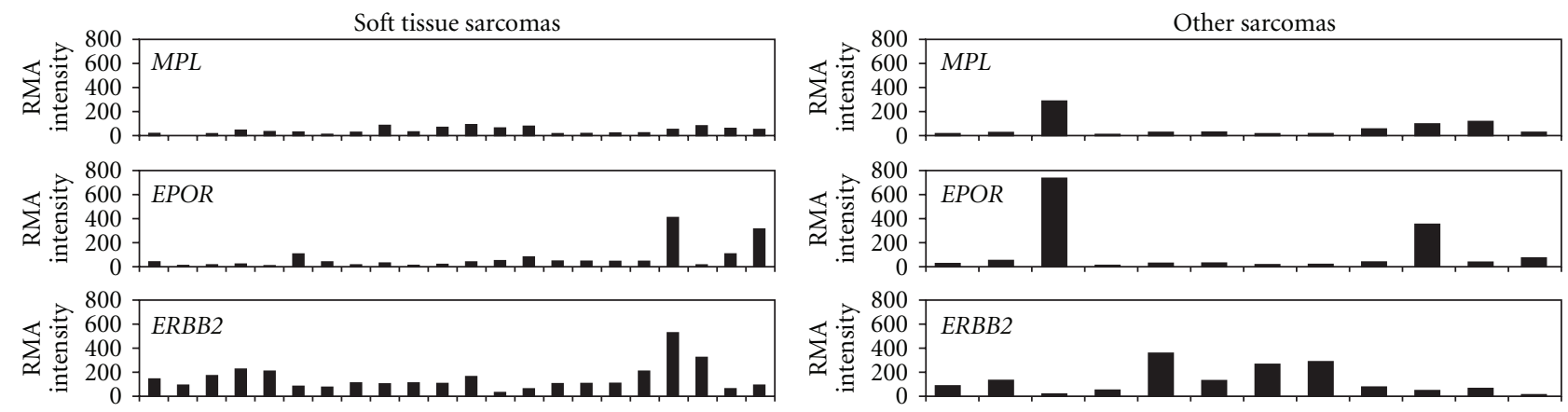

(b) Microarray data on primary sarcoma samples

Figure 2: Measurement of MPL, EPOR, ERBB2, and IGF1R mRNA in renal cell carcinoma and soft tissue and bony/cartilage sarcoma tumor samples using microarray analysis. Robust microarray average (RMA) was used for assay normalization. The levels of gene expression of $M P L, E P O R, E R B B 2$, and IGF1R mRNA were reported as RMA intensity values.

\section{Discussion}

Thrombocytopenia can be a consequence of diminished platelet production in the bone marrow, or increased platelet consumption in the spleen, liver, or circulation. Currently, platelet transfusions are used for the treatment of acute thrombocytopenia due to a variety of etiologies [27]. However, significant limitations exist to the use of platelet transfusions. Many patients become refractory to transfusion. Limited availability and cost concerns further impact the utility of platelet transfusion as an optimal treatment for thrombocytopenia. Despite donor testing and pathogen inactivation systems, infections with HIV, hepatitis $\mathrm{B}$ or $\mathrm{C}$ viruses, cytomegalovirus, or bacteria are possible. Alloimmunization and febrile transfusion reactions are 2 of the immunologically mediated adverse events that can follow platelet transfusion. And, transfusion-related acute lung injury is a potentially fatal immunologic consequence of transfusions of plasma containing blood products. Therefore, alternative treatments with fewer inherent challenges 


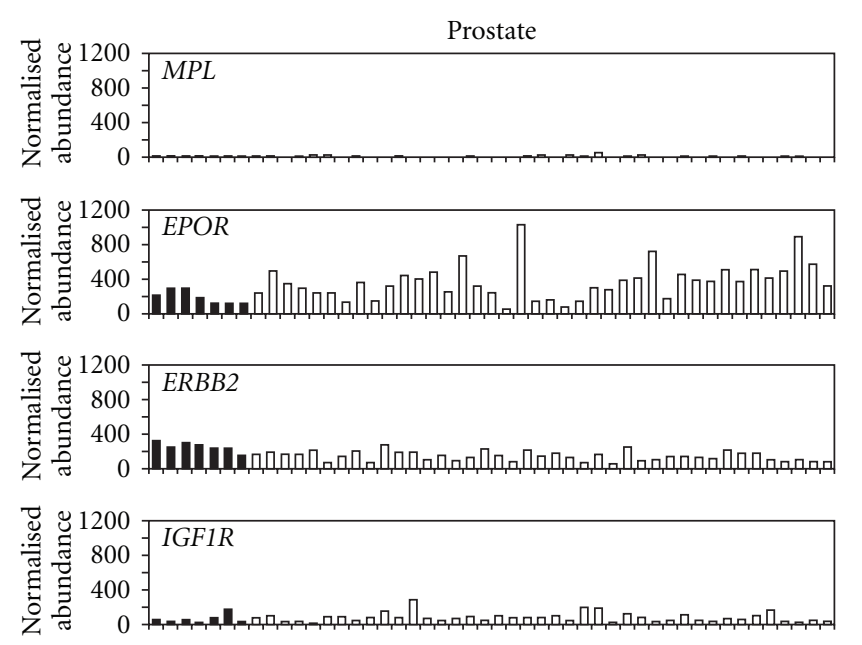

(a)
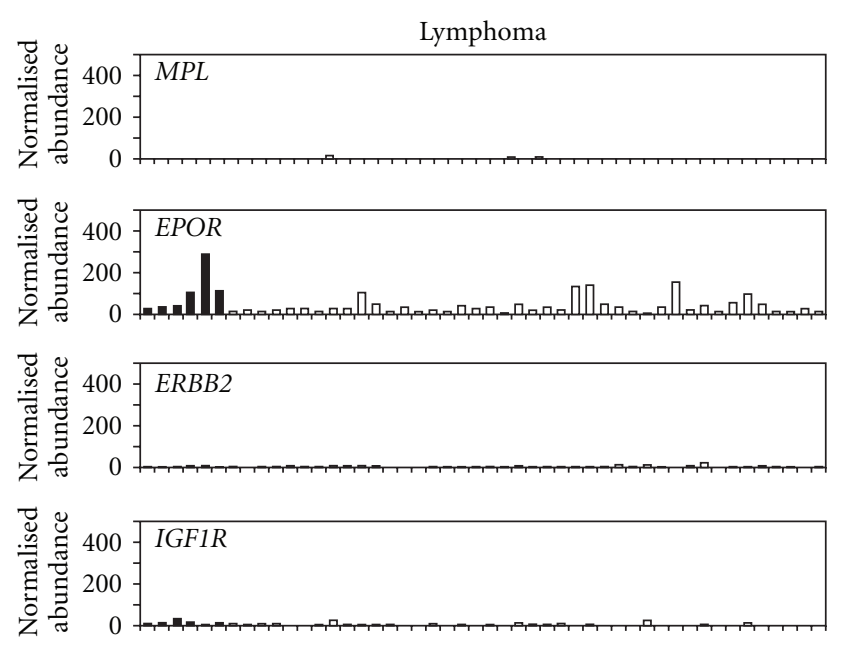

(b)
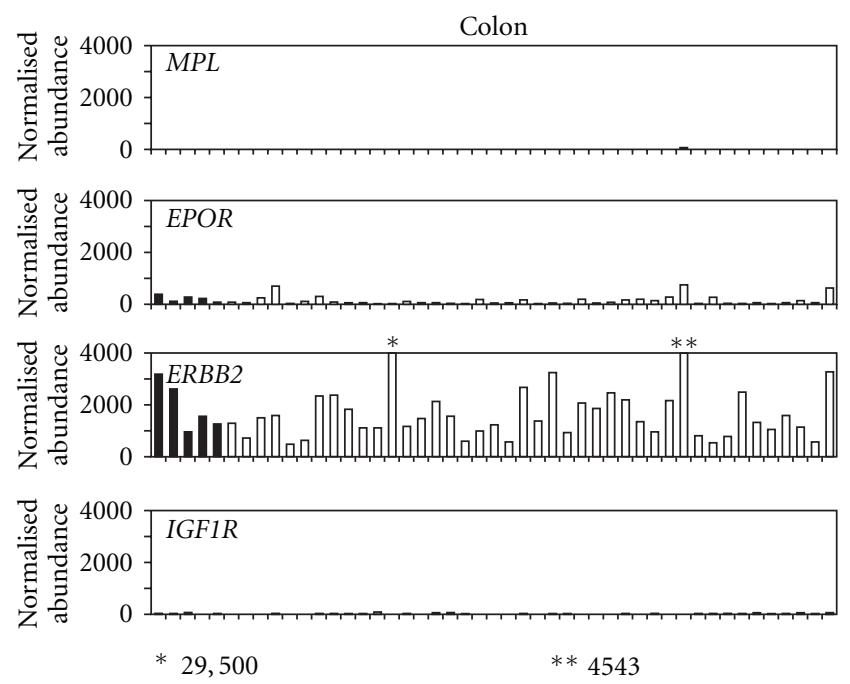

(c)

FIGURE 3: Quantitative reverse transcription-polymerase chain reaction measurement of $M P L, E P O R, E R B B 2$, and IGF1R mRNA in various normal tissue samples and tumor samples. Data were normalized to a set of housekeeping genes (GAPDH, PPIA, and ACTB). Cycle threshold $(\mathrm{Ct})$ values were calculated using the following equation: Abundance $=10 \mathrm{e}((40-\mathrm{Ct}) / 3.35)$. Samples were scaled relative to each other using the geometric mean of the set of valid housekeeping gene datapoints for that sample.

are sought. TPO-R agonists represent a class of such agents. Eltrombopag is approved for use in patients with chronic ITP and is currently in clinical trials for thrombocytopenia in patients with MDS/secondary AML and for thrombocytopenia due to chemotherapy in patients with solid tumors.

The expression of EPOR, ERBB2, and IGF1R genes in tumors has been well studied and is widely reported, thus the expression of these genes was used as a comparator for MPL [28-33].

It should be understood that expression of these genes does not always mean that the protein is detected on the cell surface. Nor does expression of a receptor protein necessarily reflect signaling or proliferation can occur $[34,35]$. The expression of TPO-R protein by Western blotting of several lung tumor cell lines, including NCI$\mathrm{H} 510$, which was one of the 3 cell lines that demonstrated high mRNA expression in this study, showed no protein expression in any of these lines [25]. Although, there are reports of AML cells-expressing MPL, 2 recent studies found no proliferation of cells in blood and marrow samples from patients with AML or MDS/secondary AML [22], or in leukemia and lymphoma cell lines in response to eltrombopag [23]; in fact, there is a decrease in proliferation at physiologically achievable concentrations of eltrombopag $[22,23]$.

Activity of TPO-R agonists depends on the expression of TPO-R in the tissue of interest. Likewise, expression of TPO-R on cells other than megakaryocytes is of great interest; activation of growth factor receptors such as TPO$\mathrm{R}$ could theoretically enhance proliferation in such tissue. The goal of this study, therefore, was to expand on the $M P L$ mRNA expression data by microarray and qRT-PCR 
previously described for breast, lung, and ovarian tumors [25] into other tumor types and tumor cell lines. MPL mRNA was consistently expressed at low or undetectable levels in the 355 tumor cell lines studied. MPL mRNA was expressed at detectable levels in 3 cell lines, the erythroleukemia lines HEL92.1.7 and KG-1, and the NCI-H510 lung tumor cell line. The HEL92.1.7 line serves as a positive control cell line in this study, as it expresses some MPL mRNA and gives a very slight, but statistically significant, proliferative response to eltrombopag [23]. A comparison of the $M P L$ mRNA expression in this line to several other leukemia cell lines, including the NOMO-1 F36-P and ML-2 cell lines, also represented in Figure 1, was previously reported [23]. In comparison, EPOR mRNA was expressed in lowto-moderate levels and ERBB2 and IGF1R mRNA were expressed at higher levels. As expected, breast tumor cell lines typically expressed much higher levels of ERBB2 mRNA. This tumor cell line data reinforced data in samples of breast and lung tumor from patients [25].

Microarray data of tumor samples revealed similar results. MPL mRNA levels were low or below the limits of detection in all tumor samples examined. The majority of tumor samples expressed mRNA for EPOR, ERBB2, and $I G F 1 R$ at varying levels. One caveat to this type of data is that the expression on normal tissues of each type are not measured. To this end, qRT-PCR of both normal tissues and tumor samples permitted a direct comparison of mRNA levels. MPL mRNA expression was low in both normal tissues and in prostate, colon, and lymphoma tumor samples, and $M P L$ mRNA was not increased in any tumor tissue relative to normal tissue.

\section{Conclusions}

Because the levels of MPL mRNA are low or undetectable in RCC, sarcoma, prostate, and colon tumors as well as lymphoma samples, it is unlikely that TPO-R agonists can induce proliferation of these types of tumor cells. Rather, TPO-R agonists will likely preferentially stimulate proliferation and differentiation of megakaryocytic cells to produce platelets and relieve thrombocytopenia. These data provide additional support for continued study of TPO-R agonists for the treatment of oncology-related thrombocytopenia and demonstrate their potential utility for correcting thrombocytopenia in clinical settings.

\section{Conflict of Interests}

C. Erickson-Miller, Y. Liu, C. Messam, K. Baker, Y. Mostafa Kamel, L. Pandite, A.-M. Martin, K. Pillarisetti, A. Chadderton, and J. Kirchner are employees of GlaxoSmithKline. As employees of GlaxoSmithKline, C. Erickson-Miller, Y. Liu, C. Messam, K. Baker, Y. Mostafa Kamel, L. Pandite, and A.M. Martin own GlaxoSmithKline stock. A. Gibbard is an employee of Morphotek. I. El-Hariry is currently employed by Astellas Pharmaceuticals, and is a former employee of GlaxoSmithKline who took part in creation of this paper.

\section{Abbreviations}

AML: $\quad$ Acute myelogenous leukemia

ATCC: American Type Culture Collection

Ct: $\quad$ Cycle threshold

DSMZ: German Collection of Microorganisms and Cell Cultures

ECACC: European Collection of Cell Cultures

EGFR: Epidermal growth factor receptor

EPOR: $\quad$ Erythropoietin receptor

ERBB2: Human epidermal growth factor

ITP: Immune thrombocytopenic purpura

IGF1R: Insulin-like growth factor-1 receptor

MDS: Myelodysplastic syndromes

mRNA: Messenger ribonucleic acid

NCI: $\quad$ National Cancer Institute

ND: $\quad$ Not determined

NSCLC: Nonsmall cell lung cancer

qRT-PCR: Quantitative reverse transcription-polymerase chain reaction

RCC: $\quad$ Renal cell carcinoma

RMA: Robust microarray average

SD: $\quad$ Standard deviation

TPO: Thrombopoietin

TPO-R: Thrombopoietin receptor.

\section{Acknowledgments}

Funding for this study was provided by GlaxoSmithKline. All listed authors meet the criteria for authorship set forth by the International Committee for Medical Journal Editors. The authors wish to acknowledge the following individuals for their contributions and critical review during the development of this paper: Rachel Brody and AOI Communications, L.P., for editorial assistance, and Kimberly Marino, of GlaxoSmithKline, for critical review.

\section{References}

[1] V. C. Broudy, N. L. Lin, and K. Kaushansky, “Thrombopoietin (c-mpl ligand) acts synergistically with erythropoietin, stem cell factor, and interleukin-11 to enhance murine megakaryocyte colony growth and increases megakaryocyte ploidy in vitro," Blood, vol. 85, no. 7, pp. 1719-1726, 1995.

[2] F. J. de Sauvage, K. Carver-Moore, S.-M. Luoh et al., "Physiological regulation of early and late stages of megakaryocytopoiesis by thrombopoietin," Journal of Experimental Medicine, vol. 183, no. 2, pp. 651-656, 1996.

[3] V. R. Deutsch and A. Tomer, "Megakaryocyte development and platelet production," British Journal of Haematology, vol. 134, no. 5, pp. 453-466, 2006.

[4] K. Kaushansky, V. C. Broudy, N. Lin et al., "Thrombopoietin, the Mpl ligand, is essential for full megakaryocyte development," Proceedings of the National Academy of Sciences of the United States of America, vol. 92, no. 8, pp. 3234-3238, 1995.

[5] D. J. Kuter and R. D. Rosenberg, "Appearance of a megakaryocyte growth-promoting activity, megapoietin, during acute thrombocytopenia in the rabbit," Blood, vol. 84, no. 5, pp. 1464-1472, 1994. 
[6] K. Peeters, J.-M. Stassen, D. Collen, C. Van Geet, and K. Freson, "Emerging treatments for thrombocytopenia: increasing platelet production," Drug Discovery Today, vol. 13, no. 17-18, pp. 798-806, 2008.

[7] P. J. Ballem, A. Belzberg, D. V. Devine et al., "Kinetic studies of the mechanism of thrombocytopenia in patients with human immunodeficiency virus infection," The New England Journal of Medicine, vol. 327, no. 25, pp. 1779-1784, 1992.

[8] S. P. Lawrence, D. C. Lezotte, J. D. Durham, D. A. Kumpe, G. T. Everson, and B. M. Bilir, "Course of thrombocytopenia of chronic liver disease after transjugular intrahepatic portosystemic shunts (TIPS). A retrospective analysis," Digestive Diseases and Sciences, vol. 40, no. 7, pp. 1575-1580, 1995.

[9] A. H. Lazarus, J. Ellis, J. W. Semple, M. Mody, A. R. Crow, and J. Freedman, "Comparison of platelet immunity in patients with SLE and with ITP," Transfusion Science, vol. 22, no. 1-2, pp. 19-27, 2000.

[10] M. Mittelman, "Platelet function in the myelodysplastic syndromes," International Journal of Hematology, vol. 71, no. 2, pp. 95-98, 2000.

[11] L. S. Elting, E. B. Rubenstein, C. G. Martin et al., "Incidence, cost, and outcomes of bleeding and chemotherapy dose modificaton among solid tumor patients with chemotherapyinduced thrombocytopenia," Journal of Clinical Oncology, vol. 19, no. 4, pp. 1137-1146, 2001.

[12] J. M. Jenkins, D. Williams, Y. Deng et al., "Phase 1 clinical study of eltrombopag, an oral, nonpeptide thrombopoietin receptor agonist," Blood, vol. 109, no. 11, pp. 4739-4741, 2007.

[13] V. C. Broudy and N. L. Lin, "AMG531 stimulates megakaryopoiesis in vitro by binding to Mpl," Cytokine, vol. 25, no. 2, pp. 52-60, 2004.

[14] C. L. Erickson-Miller, E. DelOrme, S.-S. Tian et al., "Preclinical activity of eltrombopag (SB-497115), an oral, nonpeptide thrombopoietin receptor agonist," Stem Cells, vol. 27, no. 2, pp. 424-430, 2009.

[15] J. A. Erhardt, C. L. Erickson-Miller, M. Aivado, M. Abboud, K. Pillarisetti, and J. R. Toomey, "Comparative analyses of the small molecule thrombopoietin receptor agonist eltrombopag and thrombopoietin on in vitro platelet function," Experimental Hematology, vol. 37, no. 9, pp. 1030-1037, 2009.

[16] F. Merchionne and F. Dammacco, "Biological functions and therapeutic use of erythropoiesis-stimulating agents: perplexities and perspectives," British Journal of Haematology, vol. 146, no. 2, pp. 127-141, 2009.

[17] A. M. Newland and C. D. Black, "Tumor progression associated with erythropoiesis-stimulating agents," Annals of Pharmacotherapy, vol. 42, no. 12, pp. 1865-1870, 2008.

[18] M. Wetzler, S. H. Bernstein, H. Baumann et al., "Expression and function of the megakaryocyte growth and development factor receptor in acute myeloid leukemia blasts," Leukemia and Lymphoma, vol. 30, no. 5-6, pp. 415-431, 1998.

[19] T. Motoji, M. Takanashi, S. Motomura et al., "Growth stimulatory effect of thrombopoietin on the blast cells of acute myelogenous leukaemia," British Journal of Haematology, vol. 94, no. 3, pp. 513-516, 1996.

[20] T. Murayama, S. Imoto, T. Natazuka, K. Chihara, and T. Matsui, "Proliferative reaction of myelogenous leukemia cells with cytokines G-CSF, GM-CSF, M-CSF, SCF and TPO," Leukemia Research, vol. 22, no. 6, pp. 557-560, 1998.

[21] A. Tafuri, R. M. Lemoli, M. T. Petrucci et al., "Thrombopoietin and interleukin 11 have different modulatory effects on cell cycle and programmed cell death in primary acute myeloid leukemia cells," Experimental Hematology, vol. 27, no. 8, pp. 1255-1263, 1999.
[22] B. Will, M. Kawahara, J. P. Luciano et al., "Effect of the nonpeptide thrombopoietin receptor agonist Eltrombopag on bone marrow cells from patients with acute myeloid leukemia and myelodysplastic syndrome," Blood, vol. 114, no. 18, pp. 3899-3908, 2009.

[23] C. L. Erickson-Miller, J. Kirchner, M. Aivado, R. May, P. Payne, and A. Chadderton, "Reduced proliferation of non-megakaryocytic acute myelogenous leukemia and other leukemia and lymphoma cell lines in response to eltrombopag," Leukemia Research, vol. 34, no. 9, pp. 1224-1231, 2010.

[24] I. Mavroudi, K. Pyrovolaki, K. Pavlaki et al., "Effect of the nonpeptide thrombopoietin receptor agonist eltrombopag on megakaryopoiesis of patients with lower risk myelodysplastic syndrome," Leukemia Research, 2010, In Press.

[25] C. L. Erickson-Miller, K. Pillarisetti, J. Kirchner et al., "Low or undetectable TPO receptor expression in malignant tissue and cell lines derived from breast, lung, and ovary," submitted.

[26] R. A. Irizarry, B. Hobbs, F. Collin et al., "Exploration, normalization, and summaries of high density oligonucleotide array probe level data," Biostatistics, vol. 4, no. 2, pp. 249-264, 2003.

[27] D. F. Stroncek and P. Rebulla, "Platelet transfusions," The Lancet, vol. 370, no. 9585, pp. 427-438, 2007.

[28] J. Tóvári, R. Pirker, J. Tímár, G. Ostoros, G. Kovács, and B. Döme, "Erythropoietin in cancer: an update," Current Molecular Medicine, vol. 8, no. 6, pp. 481-491, 2008.

[29] M. O. Arcasoy, "Erythropoiesis-stimulating agent use in cancer: preclinical and clinical perspectives," Clinical Cancer Research, vol. 14, no. 15, pp. 4685-4690, 2008.

[30] A. M. Sinclair, M. D. Todd, K. Forsythe, S. J. Knox, S. Elliott, and C. G. Begley, "Expression and function of erythropoietin receptors in tumors: implications for the use of erythropoiesisstimulating agents in cancer patients," Cancer, vol. 110, no. 3, pp. 477-488, 2007.

[31] H. Werner and I. Bruchim, "The insulin-like growth factor-I receptor as an oncogene," Archives of Physiology and Biochemistry, vol. 115, no. 2, pp. 58-71, 2009.

[32] A. A. Samani, S. Yakar, D. LeRoith, and P. Brodt, "The role of the IGF system in cancer growth and metastasis: overview and recent insights," Endocrine Reviews, vol. 28, no. 1, pp. 20-47, 2007.

[33] C. Sotiriou and L. Pusztai, "Gene-expression signatures in breast cancer," The New England Journal of Medicine, vol. 360, no. 8, pp. 752-800, 2009.

[34] A. M. Sinclair, N. Rogers, L. Busse et al., "Erythropoietin receptor transcription is neither elevated nor predictive of surface expression in human tumour cells," British Journal of Cancer, vol. 98, no. 6, pp. 1059-1067, 2008.

[35] M. Laugsch, E. Metzen, T. Svensson, R. Depping, and W. Jelkmann, "Lack of functional erythropoietin receptors of cancer cell lines," International Journal of Cancer, vol. 122, no. 5, pp. 1005-1011, 2008. 


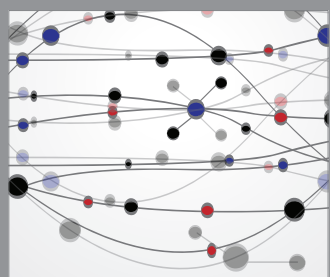

The Scientific World Journal
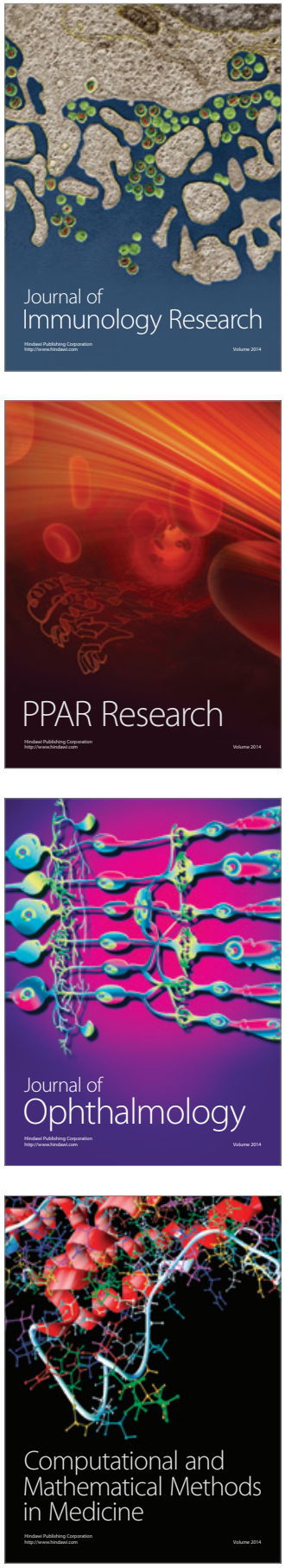

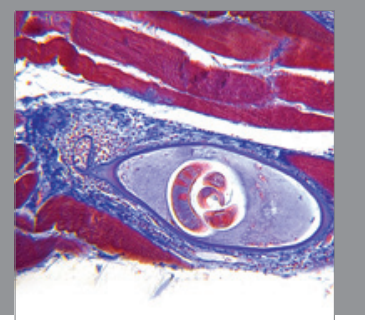

Gastroenterology

Research and Practice
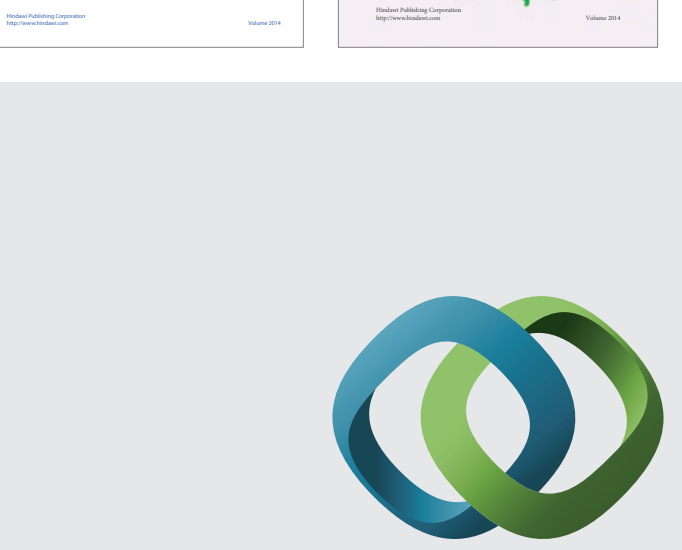

\section{Hindawi}

Submit your manuscripts at

http://www.hindawi.com
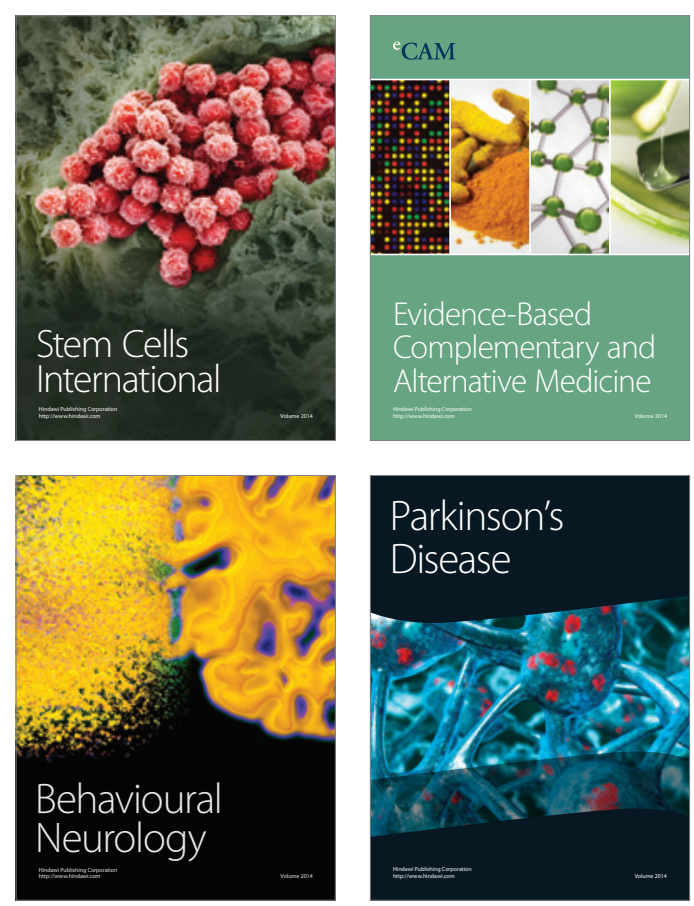

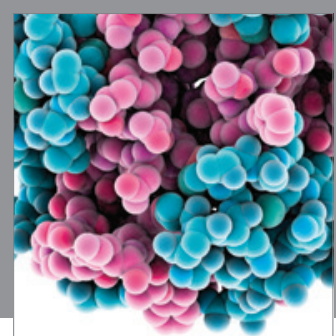

Journal of
Diabetes Research

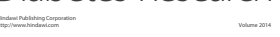

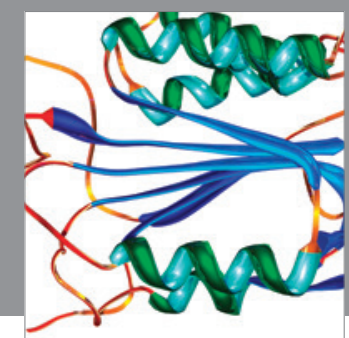

Disease Markers
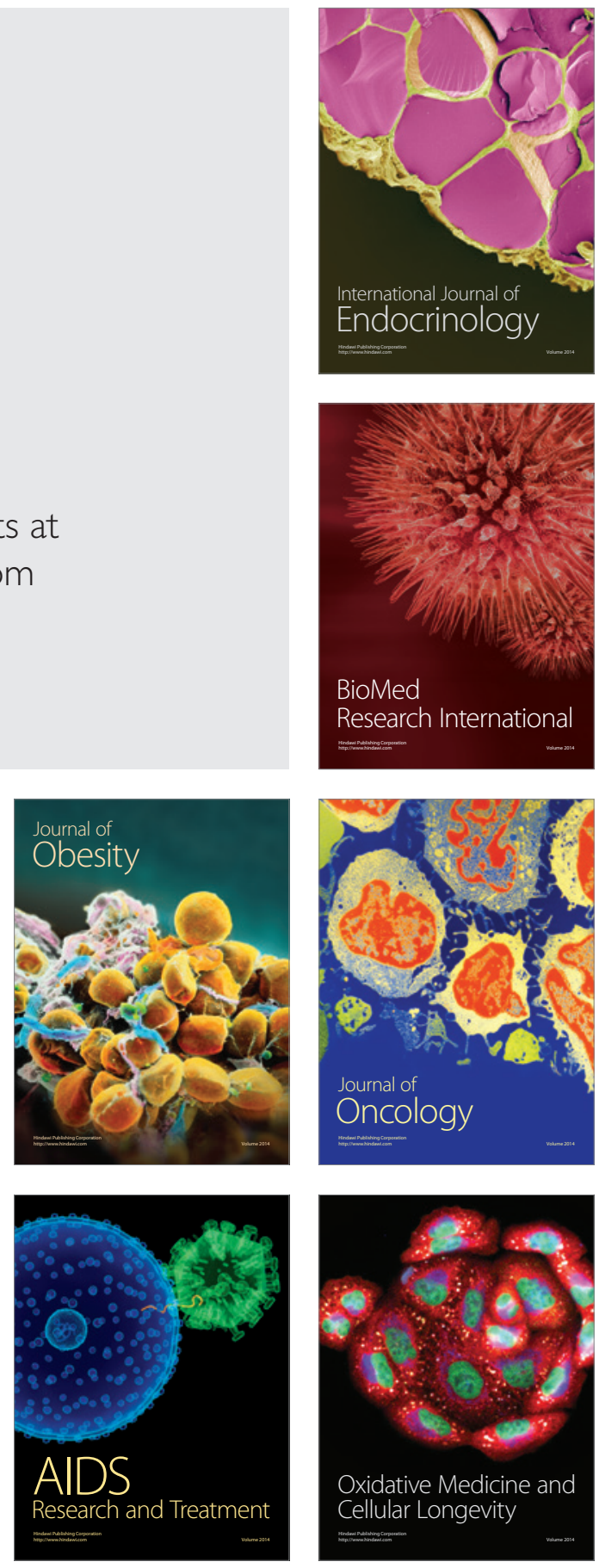